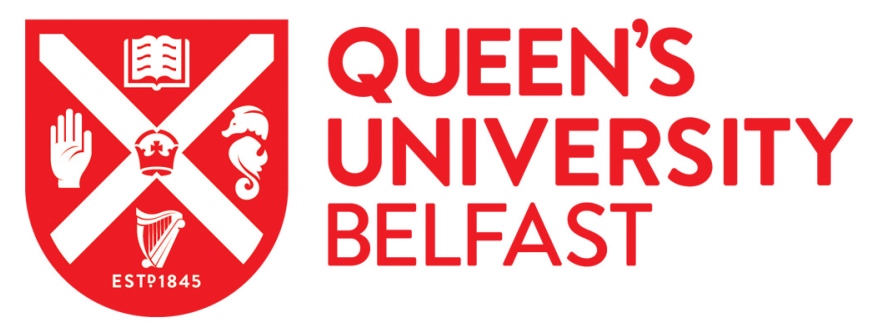

\title{
Fused Deposition Modelling as an Effective Tool for Anti-Infective Dialysis Catheter Fabrication
}

Mathew, E., Dominguez Robles, J., Stewart, S., Mancuso, E., O’Donnell, K., Larraneta Landa, E., \& Lamprou, D. (2019). Fused Deposition Modelling as an Effective Tool for Anti-Infective Dialysis Catheter Fabrication. ACS Biomaterials Science and Engineering. https://doi.org/10.1021/acsbiomaterials.9b01185

\section{Published in:}

ACS Biomaterials Science and Engineering

\section{Document Version:}

Peer reviewed version

Queen's University Belfast - Research Portal:

Link to publication record in Queen's University Belfast Research Portal

\section{Publisher rights}

Copyright 2019 American Chemical Society. This work is made available online in accordance with the publisher's policies. Please refer to any applicable terms of use of the publisher.

\section{General rights}

Copyright for the publications made accessible via the Queen's University Belfast Research Portal is retained by the author(s) and / or other copyright owners and it is a condition of accessing these publications that users recognise and abide by the legal requirements associated with these rights.

Take down policy

The Research Portal is Queen's institutional repository that provides access to Queen's research output. Every effort has been made to ensure that content in the Research Portal does not infringe any person's rights, or applicable UK laws. If you discover content in the Research Portal that you believe breaches copyright or violates any law, please contact openaccess@qub.ac.uk. 
Fused Deposition Modelling as an Effective Tool for Anti-Infective Dialysis Catheter Fabrication

Essyrose Mathew, ${ }^{1, \perp}$ Juan Domínguez-Robles, ${ }^{1, \perp}$ Sarah A. Stewart, ${ }^{1}$ Elena Mancuso, ${ }^{2}$ Kieran O'Donnell, ${ }^{2}$ Eneko Larrañeta, ${ }^{1,{ }^{*}}$ Dimitrios A. Lamprou ${ }^{1, *}$

${ }^{1}$ School of Pharmacy, Queen's University Belfast, 97 Lisburn Road, Belfast BT9 7BL, UK

${ }^{2}$ Nanotechnology and Integrated Bio-Engineering Centre (NIBEC), Ulster University, Jordanstown campus, UK

${ }^{\perp}$ These authors contributed equally to this work

\section{Corresponding authors:}

*School of Pharmacy, Queen's University Belfast, 97 Lisburn Road, Belfast BT9 7BL, UK e.larraneta@qub.ac.uk

d.lamprou@qub.ac.uk 


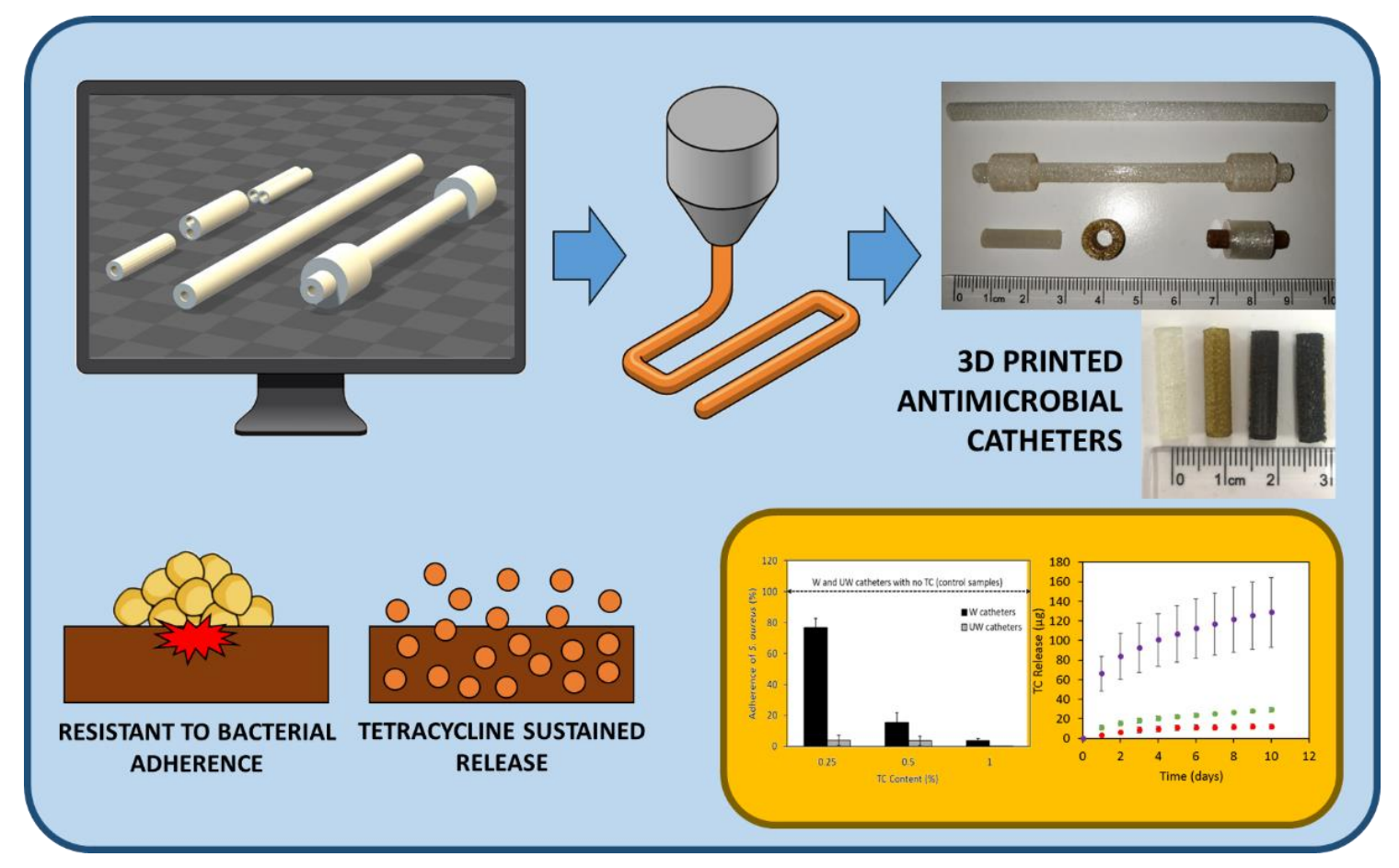

Graphical Abstract 
Abstract: Catheter associated infections are a common complication that occurs in dialysis patients. Current strategies to prevent infection include catheter coatings containing heparin, pyrogallol or silver nanoparticles, which all have an increased risk of causing resistance in bacteria. Therefore, a novel approach for manufacture, such as the use of additive manufacturing ( $A M)$, also known as 3D-printing, is required. Filaments were produced by extrusion using thermoplastic polyurethane (TPU) and Tetracycline Hydrochloride (TC) in various concentrations (e.g. $0 \%, 0.25 \%, 0.5 \%$ and $1 \%$ ). The extruded filaments were used in a fused deposition modelling (FDM) 3D-printer to print catheter constructs at varying concentrations. Release studies in phosphate buffered saline (PBS), microbiology studies, thermal analysis, contact angle, ATR-FTIR, scanning electron microscopy (SEM) and X-ray Micro Computer Tomography $(\mu \mathrm{CT})$ analysis were conducted on the printed catheters. The results suggested that TC was uniformly distributed within the TPU matrix. The microbiology testing of the catheters showed that devices containing TC had an inhibitory effect on the growth of Staphylococcus aureus NCTC 10788 bacteria. Catheters containing 1\% TC maintained inhibitory effect after 10day release studies. After an initial burst release in the first $24 \mathrm{~h}$, there was a steady release of TC in all concentrations of catheters. 3D-printed antibiotic catheters were successfully printed with inhibitory effect on $S$. aureus bacteria. Finally, TC containing catheters showed resistance to $S$. aureus adherence to their surfaces when compared with catheters containing no TC. Catheters containing $1 \%$ of TC showed a bacterial adherence reduction of up to $99.97 \%$. Accordingly, the incorporation of TC to TPU materials can be effectively used to prepare antiinfective catheters using FDM. This study highlights the potential for drug impregnated medical devices to be created through AM.

Keywords: 3D-printing; Additive Manufacturing; Fused Deposition Modelling; Extrusion; Catheters; Dialysis; Drug Release. 


\section{Introduction}

Additive manufacturing (AM), also known as 3D-printing, is an area which has gained a lot of interest in recent years due to its wide range of applications. ${ }^{1-3} 3 \mathrm{D}$-printing, allows a model which is created using computer aided design to be formed into a physical object. For this purpose, a wide variety of materials can be used, ranging from synthetic polymers to biomolecules. ${ }^{3-7}$ Due to this, AM has a wide variety of applications in diverse fields ranging from aerospace engineering to medical applications. ${ }^{4} \mathrm{AM}$ includes a wide variety of different types of techniques. All these techniques use different types of substrates such as resins, powders, gels or ceramics among others. ${ }^{8}$ However, they all have one thing in common, they produce 3D object adding material layer by layer. ${ }^{8}$ One of the most common and inexpensive types of $A M$ is fused deposition modelling (FDM). FDM requires the use of a polymeric filament that is pushed through a hot nozzle to melt the polymer and, subsequently, generating the required object. ${ }^{8}$

Diverse medical applications of 3D-printing have been described including orthopaedics, tissue engineering or the manufacture of medical devices. ${ }^{9-13}$ 3D-printing technology allows design and fabrication of structures based on images captured from patients with medical imaging techniques (i.e. magnetic resonance imaging (MRI) or computer tomography (CT)). ${ }^{3}$ Accordingly, 3D-printing can be used to prepare medical devices adapted to a particular patient's needs. Conventional fabrication techniques cannot offer this versatility.

Biocompatible materials such as poly(lactic acid) or thermoplastic polyurethane (TPU) have been used extensively for 3D-printing applications. ${ }^{4,14-16}$ This polymer very desirable for medical applications due to its resiliency, inertness in the body and blood compatibly. ${ }^{17}$ Moreover, it has been previously used for healthcare $3 \mathrm{D}$ printing applications such as the preparation of bolus for radiation therapy ${ }^{18-20}$ or the development of stents for enterocutaneous fistula surgery. ${ }^{21}$ The later work shows not only that the material is biocompatible, but also that 3D printing of TPU devices can be prepared for patient benefit.

One potential application of 3D-printing is the manufacture of medical devices such as catheters, ${ }^{22}$ as it opens up the potential for creating patient matched devices specific to their anatomy. This would allow the potential for on demand patient matched devices to be created in hospital settings. Moreover, the versatility of 3D-printing to use different types of materials can be used to add drugs, such as antibiotics, to the resulting structure. ${ }^{6,23,24}$ This is interesting for catheter manufacturing due to the tendency of these medical devices to be colonised by bacteria. A high percentage of catheter-related infections occur due to gram positive bacteria such as Staphylococcus aureus. ${ }^{25}$ This is especially problematic for dialysis catheters. 
Catheter-related infections (CRI) in dialysis patients are the second major cause of death among these patients and significantly increase the treatment cost. ${ }^{26,27}$ Staphylococcus aureus in Haemodialysis (HD) patients and Staphylococcus aureus and Pseudomonas aeruginosa in peritoneal dialysis (PD) patients are the most common causative organisms isolated. Patients with $\mathrm{CRI}$ are hospitalised more frequently than the general population, and CRI contributes to the higher associated mortality of dialysis patients compared to the general population. Considering that in the UK around 30,000 people are receiving dialysis treatment, ${ }^{28}$ it is estimated that catheter related infections of these patients are costing the UK National Health Service (NHS) ca. $£ 1.4$ million per year. ${ }^{27}$ Accordingly, the use of 3D-printing has potential to manufacture anti-infective catheters adapted to patient's needs.

In the present work, FDM will be explored for first time to our knowledge, to prepare catheters containing tetracycline (TC) to prevent bacterial colonisation of the resulting catheters. FDM works by extruding a polymer in successive layers onto a build plate where it is then cooled and solidified. For this purpose, the selected polymer was TPU, a flexible polymer that has been extensively used for biomedical applications (including catheter manufacturing). ${ }^{29-32}$ Before printing the catheters prototypes, TPU filaments containing the antibiotic were required. Accordingly, hot-melt extrusion (HME) was used to prepare these filaments. The resulting catheters were characterised by a variety of microscopic and spectroscopic techniques, and their antimicrobial properties were evaluated.

\section{Experimental Section}

\subsection{Materials}

Elastollan ${ }^{\circledR}$, thermoplastic polyurethane (TPU) elastomer pellets with 75A shore hardness were kindly provided by DistruPol Ltd (Dublin, Ireland). Tetracycline Hydrochloride (TC) drug was purchased from Sigma Aldrich, (Dorset, UK). Castor oil was obtained from Ransom (Hitchin, UK). Phosphate Buffer Solution (PBS) at pH 7.4 prepared using PBS tablets Merck (Darmstadt, Germany).

Staphylococcus aureus NCTC 10788 was maintained on cryopreservative beads in $10 \%$ glycerol at $-80^{\circ} \mathrm{C}$ and grown in Luria-Bertani (LB) broth or Mueller-Hinton Broth (MHB) at $37^{\circ} \mathrm{C}$ when required for the microbiological assessments. Moreover, to perform the different in vitro microbiological assays, several broths and solutions were used. For the zone of inhibition assay, LB agar and LB soft agar were used. Additionally, for the in vitro adherence assay Mueller-Hinton agar (MHA) and tryptone soya broth (TSB), as well as quarter-strength Ringers Solution (QSRS) and PBS were used. 


\subsection{Preparation of TC Containing TPU filaments and catheters}

HME was used to combine the drug and polymer in order to create filaments which would be used for 3D-printing. To ensure an even coating of TC on the surface of the TPU pellets, an oil method for coating was used. ${ }^{23}$ Briefly, a $50 \mathrm{~mL}$ Falcon tube was filled with TPU pellets ( $40 \mathrm{~g}$ ). Then, castor oil $(40 \mu \mathrm{L})$ was added into the tube and it was vortexed until the pellets were properly coated. Subsequently, these oil coated pellets were transferred to a new $50 \mathrm{~mL}$ Falcon tube to avoid loss of drug, by sticking to leftover oil, on the walls of tube. TC was then added in ratios of $0.25 \% \mathrm{w} / \mathrm{w}, 0.5 \% \mathrm{w} / \mathrm{w}$ and $1 \% \mathrm{w} / \mathrm{w}$ and vortexed to evenly coat all pellets. Coated pellets were added to filament extruder (3Devo, Utretch, The Netherlands) at extrusion speed of $5 \mathrm{rpm}$ and filament fan speed of $90 \%$. Finally, the temperature was adjusted through a control panel positioned at the side of the extruder and it was between 170 and $190^{\circ} \mathrm{C}$, due to the existence of 4 heaters.

\subsection{Preparation of TC Containing Catheters Using 3D-Printing.}

Once the filaments were extruded, catheters with $5 \mathrm{~mm}$ external diameter and $2 \mathrm{~mm}$ internal diameter, as well as squares of $10 \mathrm{~mm} \times 10 \mathrm{~mm} \times 1 \mathrm{~mm}$ were 3D-printed using an Ultimaker 3 (Ultimaker B.V., Geldermalsen, The Netherlands) fused filament fabrication (FFF) system and Cura ${ }^{\circledR}$ software. The Ultimaker $3 \mathrm{FFF}$ system was equipped a $0.4 \mathrm{~mm}$ nozzle. It is important to note that this equipment is a RepRap Open Source FFF equipment. ${ }^{33}$ The optimised printing parameters can be seen in Table 1.

Table 1. 3D printing parameters used for catheter production.

\begin{tabular}{ccccc}
\hline $\begin{array}{c}\text { Print-head } \\
\text { temperature }\left({ }^{\circ} \mathrm{C}\right)\end{array}$ & $\begin{array}{c}\text { Bed } \\
\text { temperature }\left({ }^{\circ} \mathrm{C}\right)\end{array}$ & $\begin{array}{c}\text { Layer height } \\
(\mathrm{mm})\end{array}$ & $\begin{array}{c}\text { Speed } \\
(\mathrm{mm} / \mathrm{s})\end{array}$ & $\begin{array}{c}\text { Infill density } \\
(\%)\end{array}$ \\
\hline 215 & 60 & 0.1 & 12 & 100 \\
\hline
\end{tabular}

\subsection{Characterisation of TPU filaments and 3D printed catheters.}

\subsubsection{Microscopy}

The morphologies of the filaments and the 3D printed catheters were assessed using a Leica EZ4 W digital microscope (Leica, Wetzlar, Germany). The microscope was equipped with florescence filters (excitation 440-460 nm and emission $500 \mathrm{~nm}$ ) (Nightsea, Lexington, MA, USA) to evaluate the distribution of TC within the filaments. Moreover, the surface morphology of the 3D-printed catheters containing TC $(0.25,0.5$ and $1.0 \%)$ and without TC, before and after the 10-days 
release study were analysed using Scanning Electron Microscope (SEM) (Hitachi TM3030, Tokyo, Japan).

\subsubsection{Mechanical properties}

TA.XTplus texture analyser (Stable Micro Systems, Surrey, UK) was used to evaluate the elastic modulus and the fracture force of the resulting filaments. Pieces of filament of $6 \mathrm{~cm}$ were cut and clamped vertically into the texture analyser. The distance between both clamps was $2 \mathrm{~cm}$. The filament was vertically stretched at a rate of $50 \mathrm{~cm} / \mathrm{min}$. The texture analyser stretched the filament up to $20 \mathrm{~cm}$. Subsequently the elastic modulus was calculated from the slope from the obtained stress/strain curves.

\subsubsection{Infrared Spectroscopy}

The Fourier Transform Infrared (FTIR) spectra of the resulting TC containing TPU materials were recorded using a Spectrum Two ${ }^{\mathrm{TM}}$ instrument (Perkin Elmer, Waltham, MA, USA) by the attenuated total reflectance (ATR) technique. The spectra were recorded from $4000 \mathrm{~cm}^{-1}$ to $600 \mathrm{~cm}^{-1}$ with a resolution of $4 \mathrm{~cm}^{-1}$ and a total of 16 scans were collected.

\subsubsection{Thermogravimetric analysis}

As the elastomer is exposed to high temperatures during the printing process, the thermal degradation behaviour of the polymer was examined. Thermogravimetric analysis (TGA) was performed to measure the weight loss of the TC containing TPU materials. For this purpose, small fragments of the filaments containing $0 \%$ and $1 \%$ TC concentration were used. TGA was performed on a Q500 Thermogravimetric analysis (TA instruments, Bellingham, WA, USA). Scans were run from room temperature to $550^{\circ} \mathrm{C}$, at a heating rate of $10^{\circ} \mathrm{C} / \mathrm{min}$ under a nitrogen flow.

\subsubsection{Contact angle}

The contact angle of water with the surface of the resulting TC containing TPU materials was evaluated using an Attension Theta equipment (Attension Theta, Biolin Scientific, Gothenburg, Sweden). OneAttension software analysed results to give an indication of the wettability of the surface.

\subsubsection{X-ray microcomputer tomography}

X-ray Micro Computer Tomography $(\mu \mathrm{CT})$ scans were also performed on 3D-printed catheters. A Bruker Skyscan 1275, with a Hamamatsu L11871 source $(40 \mathrm{kV}, 250 \mu \mathrm{A})$ and $3 \mathrm{MP}$ active pixel CMOS flat panel detector was used. Samples were mounted vertically on dental wax and positioned $59.791 \mathrm{~mm}$ from the source, where camera to source distance was $286 \mathrm{~mm}$. No filter 
was applied for an exposure time of $49 \mathrm{~ms}$. The images generated were $1944 \times 1413$ pixels with a resolution of $17 \mu \mathrm{m}$ per pixel. A total of 1056 images were taken in $0.2^{\circ}$ steps around one hemisphere of the sample, with an average of 3 frames taken at each rotation step.

The images were collected, and subsequently a volumetric reconstruction of each sample was generated using Bruker's CTvol software. Attenuation thresholding was conducted manually, in order to eliminate speckle around the samples. The same thresholding was applied within Bruker's CTan software, where the samples were further processed.

\subsection{TC release Study}

A release study was performed to calculate the amount of TC eluting from the resulting catheters. Catheters were placed in Eppendorf's containing $1 \mathrm{~mL}$ of PBS which were placed in a shaking incubator at $37^{\circ} \mathrm{C}$ and $40 \mathrm{rpm}$. Samples were taken at predetermined time intervals (each $24 \mathrm{~h}$ for 10 days). Catheters were removed from the Eppendorf's, dried and transferred to new Eppendorf's containing $1 \mathrm{~mL}$ of fresh PBS. The concentration of TC was evaluated using a UV-visible plate reader (PowerWave XS Microplate Spectrophotometer, Bio-Tek, Winooski, VT, USA) at a wavelength of $363 \mathrm{~nm}$.

\subsection{In Vitro Microbiological Analysis}

Printed catheters were tested for inhibitory effect on a bacterial culture of $S$. aureus. For this purpose, catheters at each concentration ( $0-1 \%$ of TC) were cut into $4 \times 2 \mathrm{~mm}$ discs using a scalpel. Two sets of catheters discs were tested. Catheters that were used directly after 3D-printing were referred to as unwashed (UW) catheters, and catheters that were submitted to a release in PBS media for 10 days were referred to as washed (W) catheters. Then, UW and W catheter discs were placed in a Class II Microbiological Safety Cabinet (BioMAT2) and left under UV light for $30 \mathrm{~min}$ on each side of the disc to be sterilised. To perform this experiment, $80 \mu \mathrm{l}$ of a saturated culture of $S$. aureus was added to $5 \mathrm{~mL}$ of Luria-Bertani (LB) soft agar. This mixture was then poured on top of the LB agar plate. Catheter discs were placed in the centre of the plate and incubated for 24 hours at $37^{\circ} \mathrm{C}$. The inhibition zone diameters were then measured in $\mathrm{mm}$. Furthermore, three inoculated plates with no catheter discs were also incubated as a positive control.

Additionally, an in vitro assay of bacterial adhesion onto these printed catheters was performed. For this purpose, squares (with the following dimensions: $1 \mathrm{~cm} \times 1 \mathrm{~cm} \times 0.1 \mathrm{~cm}$ ) were 3D-printed using the same filaments as for the catheters containing $0 \%, 0.25 \%, 0.5 \%$ and $1 \%$ TC. This analysis was carried out in accordance with previous published work. ${ }^{34,35}$ In brief, a bacterial 
suspension of $S$. aureus $\left(1 \times 10^{8} \mathrm{cfu} \mathrm{mL}^{-1}\right)$ in PBS and supplemented with $0.5 \% \mathrm{TSB}(\mathrm{pH} 7)$, was diluted (1:100) with PBS containing 0.5\% TSB. Replicate samples of the abovementioned 3D-printed squares were placed in individual wells of a sterile 24-well plate containing $1 \mathrm{~mL}$ of the respective bacterial suspension with a density of $1 \times 10^{6} \mathrm{cfu} \mathrm{mL}^{-1}$. The plate was continuously shaken in an orbital incubator at $37^{\circ} \mathrm{C}$ for 24 hours. The samples were then removed from the 24-well plate containing the bacterial suspension and the nonadherent bacteria were removed by serial washing, first in PBS $(1 \times 10 \mathrm{~mL})$, and secondly, in QSRS $(3 \times 10 \mathrm{~mL}) .{ }^{36}$ Samples were then transferred into fresh QSRS $(5 \mathrm{~mL})$, sonicated for $15 \mathrm{~min}$ and vortexed for $30 \mathrm{~s}$ to remove adherent bacteria. The sonication technique has previously been demonstrated not to affect bacterial viability or morphology. ${ }^{37} \mathrm{~A}$ viable count of the QSRS was performed by the Miles and Misra serial dilution technique ${ }^{38}$ followed by plating onto Mueller-Hinton agar to enumerate the previously adhered bacteria per sample. Percentage reductions in the number of adherent bacteria to each sample (UW and W catheters containing $0.25 \%, 0.5 \%$ and $1 \% \mathrm{TC}$ ) relative to the control samples (UW and W catheters containing $0 \% \mathrm{TC}$ ) were calculated.

\subsection{Statistical Analysis}

Quantitative data was expressed as a mean \pm standard deviation, $n=3$. The statistical analysis was performed using a one-way analysis of variance (ANOVA), $p<0.05$ was considered to be statistically significant.

\section{Results and Discussion}

\subsection{Preparation and Characterisation of TC Containing TPU Materials}

Extrusion of the TPU pellets containing the different TC concentrations produced smooth filaments of $2.85 \mathrm{~mm}$ in diameter (Figure 1A). Moreover, the colour of the filament darkened as the mass of TC added increased, confirming that an increased amount of TC is present in the extruded filaments. It can be inferred that the TC was mixed successfully with the TPU matrix. This can be confirmed by microscopy (Figure 1A), which show that TC was dissolved in the molten TPU within the extruder, as the filaments are homogeneous showing no visible TC aggregates. Moreover, fluorescence images did not show any TC aggregate within the material (Figure 1B). Additionally, Figure $1 \mathrm{C}$ shows the cross section of the filaments. The filaments showed homogeneous distribution of TC along the filament and accordingly this suggest that the mixing process in the extrusion was successful and that the drug and the polymer should be dissolved within the TPU matrix. Moreover, this can be confirmed by SEM, Figure S1 (supporting information). These SEM images of the cross section of blank TPU and TPU containing $1 \%$ of TC filaments showed no visible TC crystals or aggregates in the filaments. 
The good miscibility between TPU and TC can be explained by their chemical structures. The TPU used in the present work contains aromatic groups within its polyurethane units. ${ }^{39}$ On the other hand, TC contains a linear fused tetracyclic nucleus structure including a benzene ring and multiple conjugated double bonds. ${ }^{40}$ Accordingly, the interaction between the aromatic moieties of TPU and TC can explain the good miscibility between these two compounds. The interactions between TPU and TC have been previously reported by Okoli et al. ${ }^{41}$ These reported interactions were based on $\pi-\pi$ interactions between the aromatic moieties within TPU and TC. TPU filaments prepared containing TC were tested to evaluate if the presence of TC influenced their mechanical properties. Figure 1D shows the elastic modulus of the filaments as a function of the TC content. It is noticeable that the presence of TC did not alter the elastic modulus of the materials $(p>0.05)$. Moreover, the filaments were stretched up to $2000 \%$ of the initial length. None of the tested specimens fractured during the test.

To the best of our knowledge, there is no report of this type of TPU being used for 3D-printing applications. However, Christ et al. described the use of TPU Elastollan 1185A, a similar type of TPU, reinforced with carbon nanotubes for 3D-printing applications. ${ }^{42}$ The obtained elastic modulus for these filaments were higher than the ones reported here. However, this type of TPU has a higher shore hardness ( $85 \mathrm{~A}$ vs $70 \mathrm{~A}$ ) and higher inherent elastic modulus. Moreover, the addition of $1 \%$ of carbon nanotubes to the TPU significantly increased the elastic modulus by more than a $50 \%$. Interestingly, the presence of $1 \%$ of TC did not alter the elastic modulus of the filaments. Considering that TC was dissolved within the TPU during the extrusion, it can be concluded that there are interactions between the drug and the polymer. However, the results obtained suggest that the amount of drug incorporated into the polymers was not alter the mechanical properties of the material.

FTIR spectroscopy was used to evaluate if there were any interaction between TPU and TC (Figure 1E). The FTIR of TC showed peaks assigned to O-H (ca. $3460 \mathrm{~cm}^{-1}$ ), N-H (ca. $3450 \mathrm{~cm}^{-1}$ ), $\mathrm{C}=\mathrm{O}\left(1600-1700 \mathrm{~cm}^{-1}\right)$ and $=\mathrm{C}-\mathrm{N}\left(\mathrm{ca} .1460 \mathrm{~cm}^{-1}\right)$ bonds. ${ }^{43}$ On the other hand, TPU showed the characteristic peaks for the urethane group $\left(\mathrm{C}=\mathrm{O}\right.$ stretching vibration ca. $1700 \mathrm{~cm}^{-1}$ and $\mathrm{N}-\mathrm{H}$ vibrations $\left.3300-3400 \mathrm{~cm}^{-1}\right)$. ${ }^{44}$ The FTIR spectra of TPU containing $1 \%$ of TC did not any of the characteristic TC peaks or any peak shift. This could be due to the low drug content of the materials. However, it is obvious by the colour of the resulting materials (Figure $1 \mathrm{~A}-1 \mathrm{C}$ ) that the drug was blended in the TPU matrix. These results are similar to the ones obtained by Okoli et al. ${ }^{41}$ This work described the development of a TPU-based magnetic composite for TC removal from aqueous environments. In this work, the only reported changes in the FTIR spectra were 
related with the interactions between TC and the Fe within the structure. No other FTIR peaks showed changes that can suggest any interactions between TPU and TC.

FTIR spectroscopy and mechanical testing were not showing differences between TPU and TC containing materials. Accordingly, thermal analysis was performed to establish if there were any interactions between TC and TPU. TGA analysis of TPU and TPU containing 1\% of TC showed differences between these two materials (Figure 1F). The material containing TC showed an improved thermal resistance. For this purpose, the onset temperatures ( $\left.T_{\text {onset }}\right)$ for both materials were measured. Tonset denotes the temperature at which the weight loss begins. As can be seen in Figure $1 F$, the $T_{\text {onset }}$ of the TPU is shifted to a higher $\mathrm{T}_{\text {onset }}$ (from 306.5 to $322.7^{\circ} \mathrm{C}$ ) after the addition of $1 \%$ of TC (1\%). This is largely due to the existing interaction between the drug and the TPU polymeric matrix. These results suggest that there are interactions between TC and TPU. TC is preventing TPU degradation. Similar results were reported before when TPU was combined with Schiff base additives. ${ }^{44}$ These compounds acted as flame retardant when combined with TPU. Hydrogen bonding interactions of $\mathrm{O}-\mathrm{H}$ groups in the Schiff base compounds with the $\mathrm{C}=\mathrm{O}$ of the urethane group in TPU were reported. TC has multiple O-H groups capable of establishing this type of interaction.

Finally, water contact angle with TPU and TC loaded TPU materials was evaluated (Figure 1G). The obtained values ranged between 90 and $105^{\circ}$. Accordingly, it can be established that all the prepared materials were hydrophobic in nature as water contact angle values were higher than $90^{\circ} .{ }^{45}$ No significant differences were obtained between all the TC containing TPU materials $(p>0.05)$. However, the contact angle showed lower values when the TC concentration was increased from 0.5 to $1 \%$. In the present study, TC was used in its hydrochloride salt form. Accordingly, this drug is a hydrophilic drug. This explains why materials containing higher TC loadings showed "lower" hydrophilicity. 

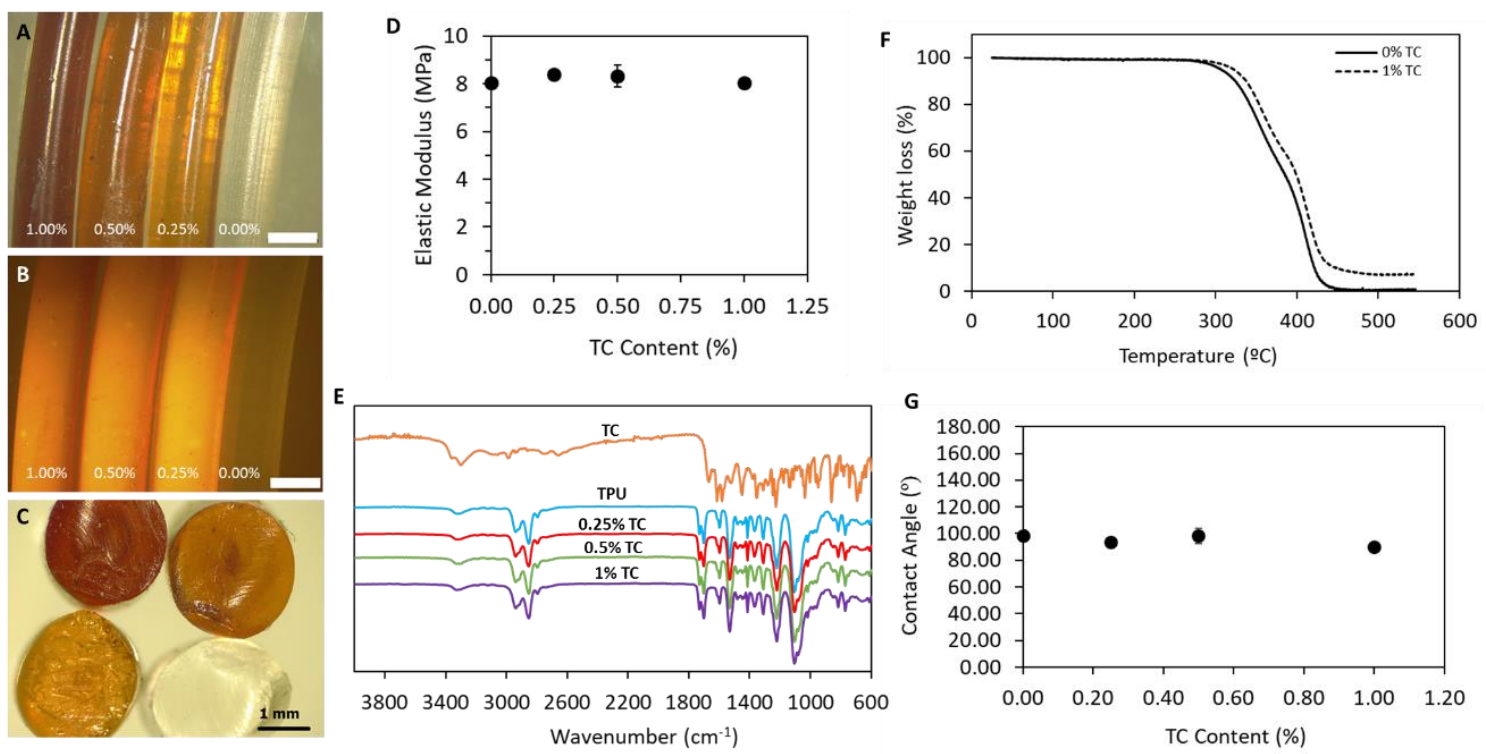

Figure 1 (A). Microscopy (A) and fluorescent microscopy (B) images of TPU filaments containing $0 \%, 0.25 \%, 0.5 \%$ and $1 \%$ of TC. Cross section microscopy image of the filaments (C). Elastic modulus of the TC containing filaments (D). FTIR-ATR of TC containing TPU materials (E). TG curves TC containing TPU materials ( $0 \%$ and $1 \%$ TC) (F). Contact angle of water with the surface of TC containing TPU materials (G).

\subsection{Preparation of TC Containing Catheters Using 3D-Printing.}

TPU and TPU/TC filaments were used to produce catheters using fused deposition modelling. Figure 2 shows fragments of catheters produced with the previously mentioned materials. It can be seen that catheters containing higher TC loadings showed darker colours (Figure 2A). Moreover, due to the inherent flexibility of TPU the resulting catheters were flexible (Figure 2B and 2 C). Central venous lines require the presence of cuffs to provide stability once they are in place. Figure 2D shows that the technique can be used to prepare more sophisticated catheters such as cuffed catheters. Moreover, FDM can be used to prepare independent cuffs with and without antibiotic (Figure 2D). The development of antiseptic cuffs to prevent catheter-related infections has been previously described. ${ }^{46}$ However, the literature report that the most used bactericidal agent for this purpose was silver and it showed limited effectivity. ${ }^{47,48}$ Accordingly, we believe that the use of antibiotic, such as TC, will significantly reduce bacterial colonisation of the material. The results showed in the present paper are a proof of concept and, accordingly, the catheters used for further studies were on lumen catheters. However, catheters used for HD or central venous lines present more complex designs including more than one lumen. ${ }^{49}$ Figure $2 \mathrm{E}-2 \mathrm{G}$ shows that FDM can be used to prepare these types of designs. Finally, it is important to note that liquid can flow through the catheters without leaking. To illustrate this a methylene 
blue solution was injected trough a 3D printed catheter (Figure $2 \mathrm{H}$ ). During the process, no leakage was observed.

The use of 3D-printing catheters has been described before. ${ }^{22,24}$ However, these publications described the concept and showed some examples of catheters prepared using poly(lactic acid) (PLA), ${ }^{23,24}$ a commonly used 3D-printing material. This is not a good material for this application as full catheters cannot be produced using this material as it is not flexible. Realistically, these catheters cannot be used in patients. ${ }^{22}$ The catheters described here are flexible (Figure 2) as they are made of TPU. This is the same material that is used to prepare catheters and central venous lines. ${ }^{50}$ Moreover, FDM allows the on demand preparation of catheters and adaption to the needs of the patient. This is especially interesting for children that require catheters with non-conventional sizes. ${ }^{51}$
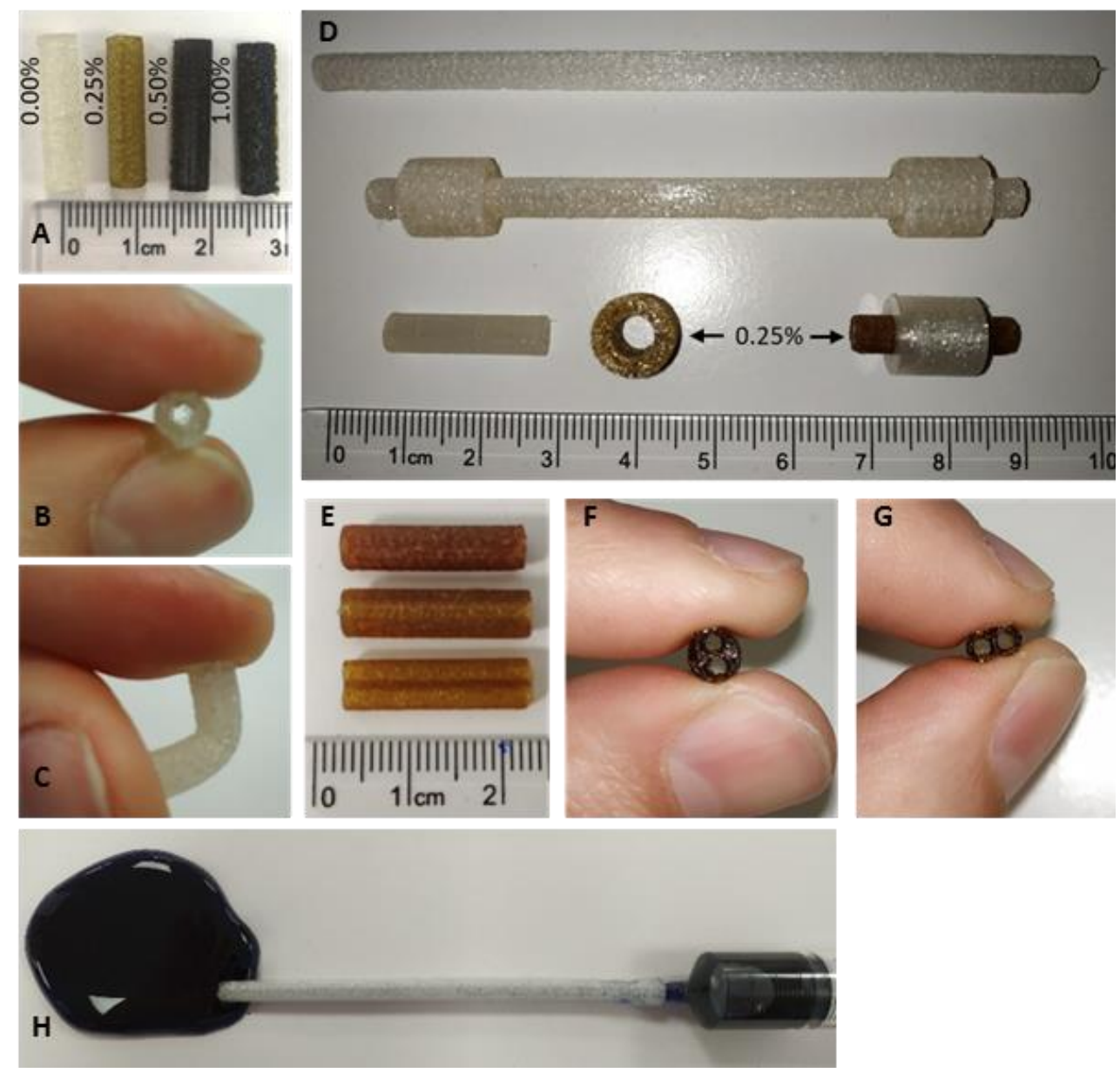

Figure 2. Catheters produced by Ultimaker3 Printer at concentrations of $0 \%, 0.25 \%, 0.5 \%$ and $1 \%$ TC showing their flexibility (A, B and C). Longer catheters with and without integrated cuffs, and spare printed cuffs with and without antibiotics (D). Different designed catheters used for dialysis and central venous lines (with two lumens) (E). Injection of a methylene blue solution $(0.5 \mathrm{mg} / \mathrm{mL})$ through a TPU catheter. 
SEM was used to characterise the surface of the printed catheters. These images show smooth uniform layers in the images before release (Figure 3). As can be seen in the Figure 3, there were no visible modifications in the layered structure of the catheters after the release study. FDM is an $\mathrm{AM}$ technique that creates 3D objects by sequential layer deposition. ${ }^{52}$ SEM images showed the layers of the catheters printed using this technique. No differences are observed between blank catheters and TC loaded catheters.

The surface of the catheter's present certain degree of roughness and some minor defects. However, the prepared catheters did not leak as shown in Figure $2 \mathrm{H}$. The work described here is a proof of concept and more work need to be developed before these materials can be used in real patients. One of the key aspects that can be improved for future developments is the surface roughness. There are different techniques that can be applied to improve it. The easiest one is use solvent vapour to smooth the surface of the prints. ${ }^{53}$ However, this adds an additional step to the process and it is not ideal. An alternative to this is to implement alternative ways of printing. Nonplanar 3D printing can be used to obtain smoother surfaces. ${ }^{54,55}$ This is not a new type of 3D printing but a new software algorithm to treat computer generated objects before printing. Accordingly, this novel software technique can be implemented in conventional FDM printers.
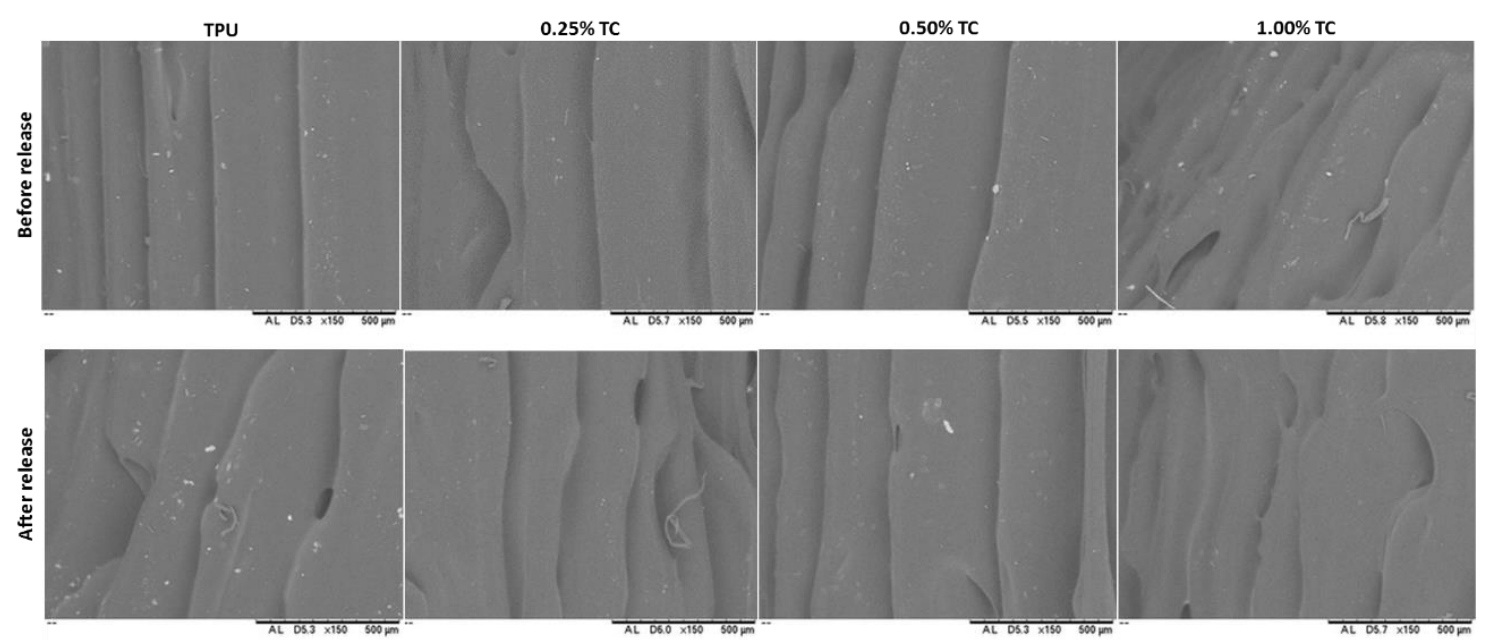

Figure 3. SEM micrography of 3D-printed catheters at concentrations of $0 \%, 0.25 \%, 0.5 \%$ and $1 \%$ before and after the release study. The scale bar represents $500 \mu \mathrm{m}$.

The 3D-printed sample architectures and topologies were analysed through the Bruker Skyscan 1172 system $\mu \mathrm{CT}$. As it could be seen in the 3D volume reconstruction (Figure S2 in the supplementary material section), performed by using the Bruker's CTvol software, the drug incorporation did not affect the morphology of the 3D-printed samples, which were very similar 
for all TC concentrations. In addition, even at the highest drug concentration (Figure S2E-S2F) all the samples were characterised by a continuous outer shell. In accordance to other studies published in the literature, ${ }^{56-58}$ and where similar manufacturing processes were applied, this might be due the different dimensional range between the polymer, acting as a matrix, and the drug.

The representative images of the samples' cross sections are shown in Figure 4. It has been found that the topology of control specimens (Figure 4A-4B) was comparable to those samples characterised by a low drug dose (see Figure 4C-4D). The presence of the drug particles was more evident in the samples loaded with $1 \%$ of TC, as can be seen in Figure $4 \mathrm{E}$, and for which the washing process did not affect the drug retention (Figure 4F).
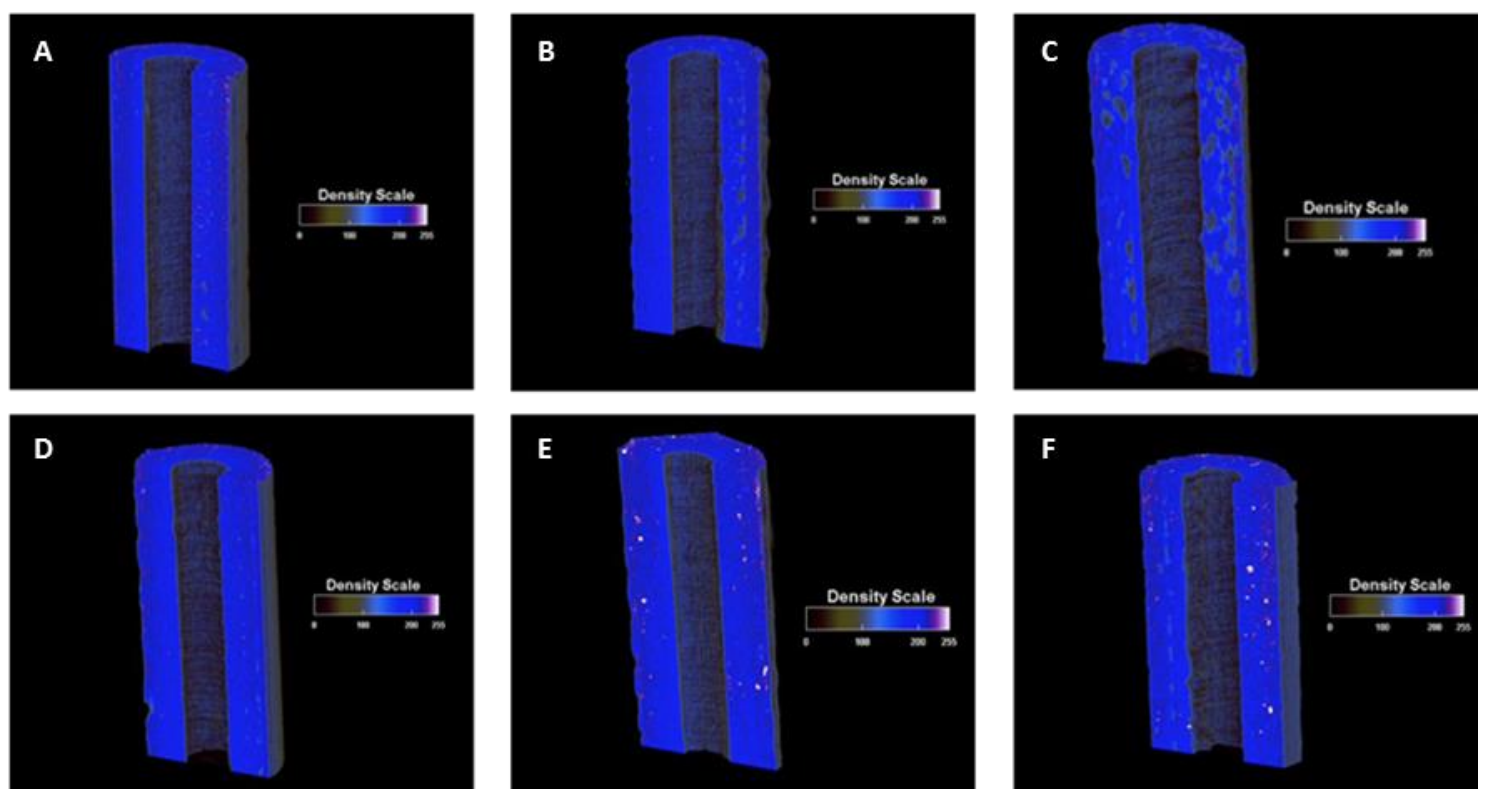

Figure 4. $\mu C T$ cross section images of $0 \%$ TC W catheter (A) $0 \%$ TC UW catheter (B) $0.25 \%$ TC UW catheter (C) $0.5 \%$ TC UW catheter (D) 1\% TC UW catheter (E) and 1\% TC W catheter (F).

Furthermore, it is possible to appreciate the distribution of the drug within the 3D structure (Figure 5). The presence of the drug in each one of the 3D-printed specimens was assessed by further analysis and 3D reconstructions. As reported in Figure 5, the distribution of the drug within the 3D volumes was uniform and proportional to the concentration loaded. Moreover, even in this case it was demonstrated that the release step (Figure 5F) did not affect the drug retention, if compared to the UW catheter (Figure 5E). 

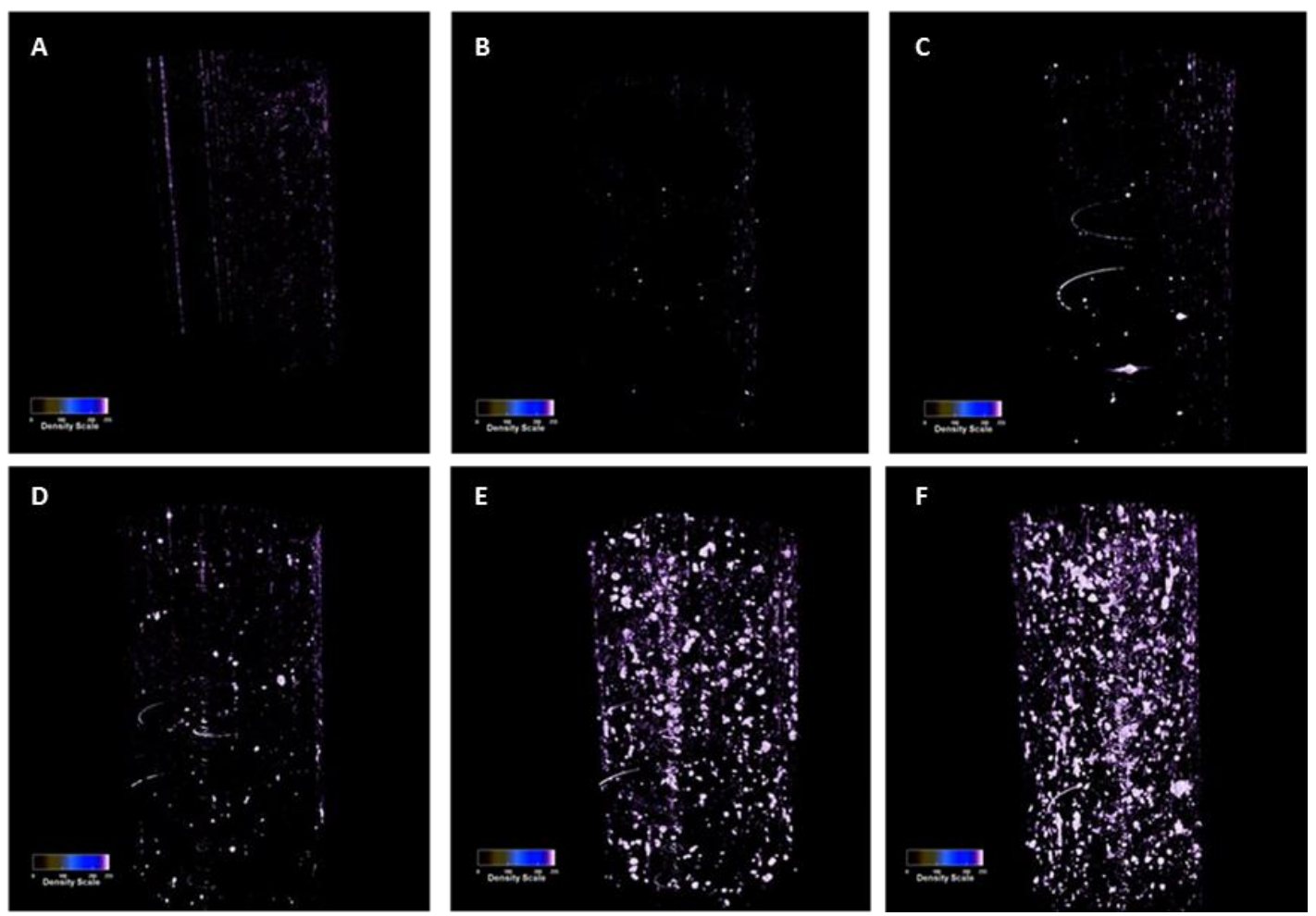

Figure 5. 3D reconstruction of the drug distribution in the 3D-printed volume of the $0 \%$ TC W catheter (A) 0\% TC UW catheter (B) 0.25\% TC UW catheter (C) 0.5\% TC UW catheter (D) 1\% TC UW catheter (E) and 1\% TC W catheter (F).

\subsection{Release Studies}

Drug release from catheter structures was analysed using UV analysis. A similar pattern of release was followed by the concentration of drug at $0.25 \%$ TC and $0.5 \%$ TC (Figure 6). There is an initial burst release of TC released in the first day and then the amount released begins to plateau after the third day. However, catheters containing $1 \%$ of TC had a much higher amount of TC released initially compared to the previous ones $(p<0.05)$. At the end of the ten days, there is still an increasing level of TC release for the catheters containing $1 \%$ of TC. Although these catheters had a much larger release of TC, this is was around $4 \%$ release of the total loaded drug after ten days. 


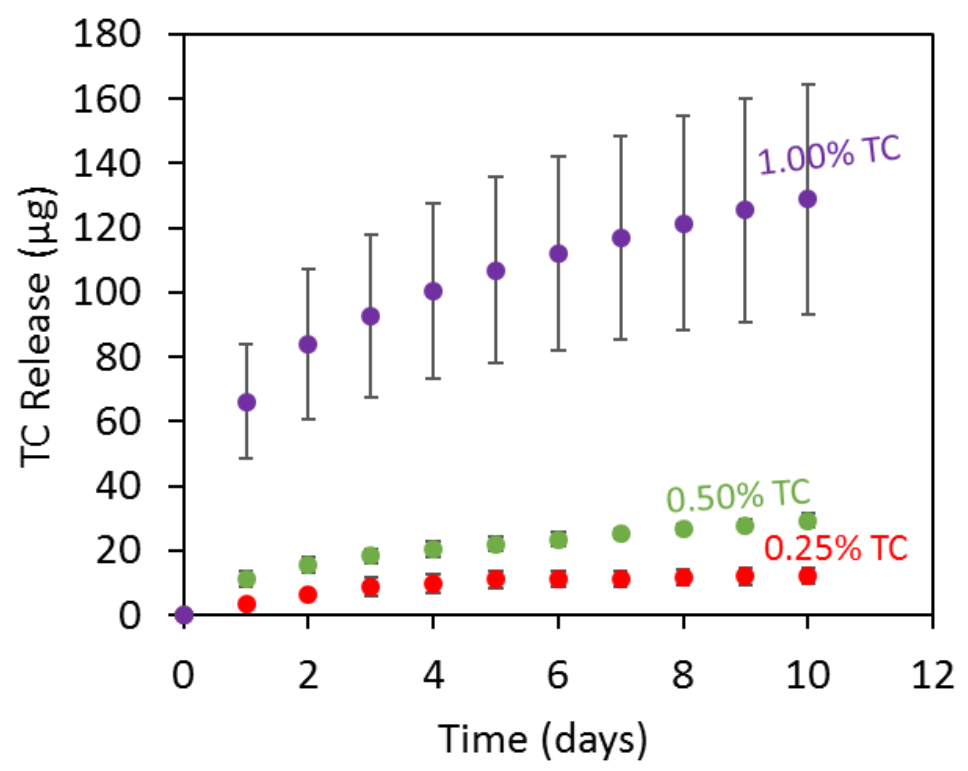

Figure 6. Cumulative TC release from 3D-printed catheters containing $0.25 \%, 0.5 \%$ and $1 \%$ of TC (Means \pm S.D; $\mathrm{n}=3$ ).

The initial burst release of drug from all of the catheters may be due to the presence of TC on the surface of the catheters. The TC present in the outermost layers of the catheter constructs would have a smaller diffusion distance into the surrounding medium and so may have caused the initial burst release of drug. The abovementioned PLA catheters containing gentamicin sulphate (GS) or methotrexate (MTX) showed a similar pattern to the present study, an initial burst release during the first few hours followed by a steady release. ${ }^{24}$

After ten days, the catheter containing $1 \%$ of TC was still releasing and only $4 \%$ of the drug was released. This sustained drug release profile may be due to the hydrophobic nature (Figure 1G) of the TPU polymer, which would therefore slow water penetration into polymer matrix. Therefore, this catheter has potential to have prolonged antibacterial effects, as it will be discussed in the following section.

\subsection{In Vitro Microbiological Analysis}

Printed catheters were tested for antimicrobial effect on a bacterial culture of $S$. aureus. UW catheters and $\mathrm{W}$ catheters were used for this assay. The results of the zone of inhibition are presented in the Figure 7A-7B. A zone of inhibition indicates that $S$. aureus either at the surface of the catheters or even for an area extending outwards form the catheters surface are inhibited. Both, UW and W catheters containing TC exhibited zones of inhibition. As expected, the control catheters containing no TC had no zones of inhibition. The zones of inhibition were increased by increasing the amount of TC. However, statistical analysis showed that there were no significant 
differences between the zones of inhibition caused by UW catheters containing $0.25 \%$ and $0.5 \%$ TC, and the same was observed for $\mathrm{W}$ catheters at the same concentrations. $(p>0.05)$. Therefore, it can be inferred that increase in TC concentration from $0.25 \%$ to $0.5 \%$ did not have a significant impact on the zones of inhibition produced. However, there was a clear increase in the zone of inhibition from $0.25 \%$ to $1 \%(p<0.05)$ for both UW and W catheters.

On the other hand, comparing UW with W catheters, at $0.25 \%$ and $0.5 \% \mathrm{TC}$ concentrations, there was a decrease in the zones of inhibition after ten days releasing TC in PBS $(p<0.05)$. The average zone of inhibition for $\mathrm{W}$ catheters at $0.25 \%$ and $0.5 \%$ TC concentrations were 6.0 and $7.6 \mathrm{~mm}$, respectively, compared to $9.3 \mathrm{~mm}$ and $11.5 \mathrm{~mm}$ before the releasing step (UW catheters). Therefore, less TC is diffusing out of the W catheters compared to the UW ones. Nevertheless, there was no significant differences between the halos found for both UW and W catheters at $1 \%$ TC $(p>0.05)$, which is in line with the obtained drug release profile for the catheters containing $1 \%$ of TC.
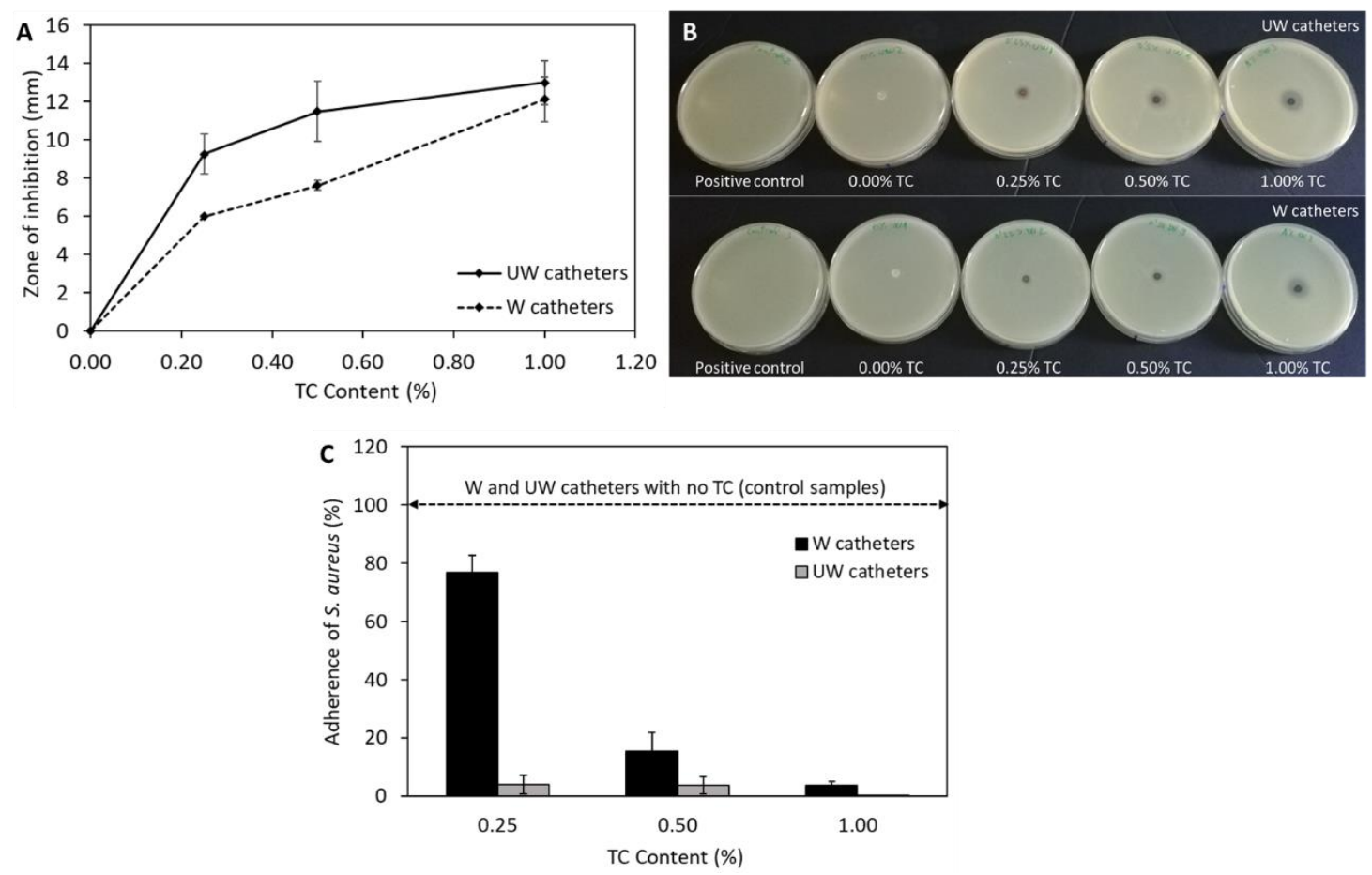

Figure 7. Correlation between the diameter of the inhibition zone of $S$. aureus and the concentration of TC (A). Positive S. aureus control and agar plates showing zones of inhibition of UW and W catheters (B). Microbial adherence (\%) of S. aureus to UW and W catheters after $24 \mathrm{~h}$ at $37^{\circ} \mathrm{C} p($ HEMA) and copolymers of 2-HEMA and conjugates1-3relative to the p(HEMA) homopolymer, denoted control, after 4 and $24 \mathrm{~h}$ incubation at $37^{\circ} \mathrm{C}$. Columns and error bars represent means $\pm S D(n=3)$. 
It is clear that the catheters had bacteriostatic activity on the $S$. aureus culture (Figure 7A-7B). This further supports the hypothesis that extrusion and 3D-printing did not affect the bacteriostatic activity of this drug. Catheters containing $0.25 \%$ and $0.5 \%$ TC had lower bacteriostatic activity after the ten-day releasing step. This is due to the release of TC during this step, which would have resulted in the majority of drug on the outer layers of the catheters to have diffused out. Furthermore, catheters containing 1\% TC still had significant bacteriostatic activity after the release study. It matched the conclusions of this release study as drug was still being eluted from the catheter after ten days. This drug concentration (1\%) is relatively low, however, it was still able to produce significant zones of inhibition. An issue that exists with current coated catheters is the risk of toxicity. The ability to use such small quantities of drug and still have bacteriostatic activity minimises the risk of these drug impregnated catheters causing toxicity in the patient. Genina et $a{ }^{59}$ reported the possibility to print drug-loaded intrauterine devices using different grades of ethylene vinyl acetate (EVA) and higher percentage of drugs ( 5 and $15 \%$ of indomethacin). Weisman et al. ${ }^{23}$ conducted a study on 3D-printed antibiotic and chemotherapeutic constructs in which $1 \%$ and $2.5 \%$ gentamicin was used. The $1 \%$ disc tested in bacterial culture of $E$. coli had a similar zone of inhibition $(12.9 \mathrm{~mm})$ to $1 \% \mathrm{TC}$ catheter in this study $(13.0 \mathrm{~mm})$. It is well known that these results depend on the bacteria strain used, the amount of it, as well as the type of drug. For instance, in this study $80 \mu \mathrm{l}$ of a S. aureus culture were used compared with the $50 \mu \mathrm{l}$ used in the previous work. ${ }^{23}$

In addition to the zone of inhibition assay, another in vitro assay to study the capacity to avoid a biofilm formation on the part of the coated catheters was performed. Bacterial adherence to the surface of the catheters was studied with a model of opportunistic pathogen as S. aureus. ${ }^{60}$ Moreover, S. aureus is a common causative agent of medical device and bloodstream infections. ${ }^{61}$

The results of the efficacy of these catheters in resisting adherence of $S$. aureus relative to the control catheters containing no TC are presented in the Figure $7 C$. The greatest reduction in adherence of $S$. aureus was achieved by UW catheters containing $1 \%$ TC with adherence reduced by a mean value of $99.97 \%$ after 24 hours (Figure $7 C$ ). Nevertheless, this excellent reduction value is followed closely by the UW catheters containing $0.25 \%$ and $0.5 \% \mathrm{TC}$ as well as the W catheters containing $1 \%$ TC, which showed reduction in bacterial adherence values of 96.01 , 96.37 and $96.33 \%$ after $24 \mathrm{~h}$, respectively. These results agreed with those of studies on zone of inhibition. W catheters containing $1 \%$ TC were able to avoid a biofilm formation even after ten days releasing TC. Indeed, there were not significant differences between all the UW catheters 
and the W catheter containing 1\% TC $(p>0.05)$. Moreover, even W catheters containing $0.5 \%$ TC (after ten days releasing TC) showed also good results, in terms of reduction values (84.62\%), similar to those obtained using nalidixic acid. ${ }^{35}$ Only W catheters containing small amounts of TC (0.25\%) showed poor reduction values (23.26\%). These results are therefore extremely promising considering that antibacterial materials have potential to be used for biomedical applications.

'Nosocomial' or 'healthcare associated infections' (HCAI) affect patients under medical care in the different health care facilities, such as hospitals. ${ }^{62}$ These infections can occur through the use of medical devices such as catheters and ventilators employed in modern health care. ${ }^{63}$ According to The World Health Organization (WHO), these infections could affect approximately $15 \%$ of all hospitalized patients. ${ }^{64}$ Moreover, these infections can be caused by bacteria, viruses and fugal parasites, however, bacteria are the most common pathogens responsible for HCAl. Highly resistant bacteria such as Methicillin-resistant $S$. aureus are the cause of large part of these infections worldwide. This means long stays in the health care facilities while increasing health care costs. ${ }^{65}$ Therefore, these novel 3D-printed antibacterial devices, such as catheters, have a great potential to minimise the appearance of these HCAl.

\section{Conclusions}

This study demonstrates the potential to incorporate drugs into the 3D-printed manufacture of medical devices. TC was combined with TPU using single screw HME to yield filaments with the drug dissolved in the matrix. This was confirmed by SEM and TGA measurements. Moreover, the addition of up to $1 \%(\mathrm{w} / \mathrm{w})$ of TC did not influence the mechanical or surface properties of the resulting material. These filaments were used to successfully prepare catheters via-FDM.

TC containing catheters were capable of releasing TC for periods of up to 10 days. Moreover, the drug loaded TC catheters had an inhibitory effect on S. aureus bacteria, which commonly causes catheter related infections. This was especially significant for $1 \%(w / w)$ catheters. The tested catheters showed inhibitory effect on $S$. aureus bacteria even after being washed for 10 days in PBS. However only catheters containing $1 \%(w / w)$ of TC showed equivalent inhibitory effect before and after 10 days of washing. Finally, TC catheters showed resistance to bacterial adherence when compared with TPU catheters (up to $99.97 \%$ reduction).

These results showed the potential of FDM for anti-infective catheter manufacturing. This is only one potential application of this technology. This proof of concept study displays how antibacterial catheters can be created to combat catheter related infections associated with 
dialysis catheters. This is the first study that describes the production of usable anti-infective catheters using 3D-printing.

The methods described in the present paper can be applied to various medical devices and a range of different drug eluting materials. Further research should be done such as the in vivo evaluation of the catheters to fully test their bioactivity and adverse effects. Moreover, important aspects such as the sterilisation of such devices should be evaluated. Finally, there are regulatory questions still open about the use of 3D-printing for medical device production. However, the US Food and Drug Administration (FDA) has started investigate this potential technology and has published guidelines for manufacturers on how to use this technology appropriately. ${ }^{66}$

\section{References:}

(1) Hart, L. R.; Li, S.; Sturgess, C.; Wildman, R.; Jones, J. R.; Hayes, W. 3D Printing of Biocompatible Supramolecular Polymers and Their Composites. ACS Appl. Mater. Interfaces 2016, 8 (5), 3115-3122. https://doi.org/10.1021/acsami.5b10471.

(2) Palaganas, N. B.; Mangadlao, J. D.; de Leon, A. C. C.; Palaganas, J. O.; Pangilinan, K. D.; Lee, Y. J.; Advincula, R. C. 3D Printing of Photocurable Cellulose Nanocrystal Composite for Fabrication of Complex Architectures via Stereolithography. ACS Appl. Mater. Interfaces 2017, 9 (39), 34314-34324. https://doi.org/10.1021/acsami.7b09223.

(3) Guvendiren, M.; Molde, J.; Soares, R. M. D.; Kohn, J. Designing Biomaterials for 3D Printing. ACS Biomater. Sci. Eng. 2016, 2 (10), 1679-1693. https://doi.org/10.1021/acsbiomaterials.6b00121.

(4) Ligon, S. C.; Liska, R.; Stampfl, J.; Gurr, M.; Mülhaupt, R. Polymers for 3D Printing and Customized Additive Manufacturing. Chem. Rev. 2017, 117 (15), 10212-10290. https://doi.org/10.1021/acs.chemrev.7b00074.

(5) Sommer, M. R.; Schaffner, M.; Carnelli, D.; Studart, A. R. 3D Printing of Hierarchical Silk Fibroin Structures. ACS Appl. Mater. Interfaces 2016, 8 (50), 34677-34685. https://doi.org/10.1021/acsami.6b11440.

(6) Domínguez-Robles, J.; Martin, N.; Fong, M.; Stewart, S.; Irwin, N.; Rial-Hermida, M.; Donnelly, R.; Larrañeta, E. Antioxidant PLA Composites Containing Lignin for 3D Printing Applications: A Potential Material for Healthcare Applications. Pharmaceutics 2019, 11 (4), 165. https://doi.org/10.3390/pharmaceutics11040165. 
(7) Jose, R. R.; Rodriguez, M. J.; Dixon, T. A.; Omenetto, F.; Kaplan, D. L. Evolution of Bioinks and Additive Manufacturing Technologies for 3D Bioprinting. ACS Biomater. Sci. Eng. 2016, 2 (10), 1662-1678. https://doi.org/10.1021/acsbiomaterials.6b00088.

(8) Tofail, S. A. M.; Koumoulos, E. P.; Bandyopadhyay, A.; Bose, S.; O’Donoghue, L.; Charitidis, C. Additive Manufacturing: Scientific and Technological Challenges, Market Uptake and Opportunities. Mater. Today 2018, $21 \quad$ (1), 22-37. https://doi.org/10.1016/j.mattod.2017.07.001.

(9) Murphy, S. V; Atala, A. 3D Bioprinting of Tissues and Organs. Nat. Biotechnol. 2014, 32 (8), 773-785. https://doi.org/10.1038/nbt.2958.

(10) Zhao, H.; Yang, F.; Fu, J.; Gao, Q.; Liu, A.; Sun, M.; He, Y. Printing@Clinic: From Medical Models to Organ Implants. ACS Biomater. Sci. Eng. 2017, 3 (12), 3083-3097. https://doi.org/10.1021/acsbiomaterials.7b00542.

(11) Jang, J.; Yi, H.-G.; Cho, D.-W. 3D Printed Tissue Models: Present and Future. ACS Biomater. Sci. Eng. 2016, 2 (10), 1722-1731. https://doi.org/10.1021/acsbiomaterials.6b00129.

(12) Sandler, N.; Salmela, I.; Fallarero, A.; Rosling, A.; Khajeheian, M.; Kolakovic, R.; Genina, N.; Nyman, J.; Vuorela, P. Towards Fabrication of 3D Printed Medical Devices to Prevent Biofilm Formation. Int. J. Pharm. 2014, 459 (1-2), 62-64. https://doi.org/10.1016/j.ijpharm.2013.11.001.

(13) Gibbs, D. M.; Vaezi, M.; Yang, S.; Oreffo, R. O. Hope versus Hype: What Can Additive Manufacturing Realistically Offer Trauma and Orthopedic Surgery? Regen. Med. 2014, 9 (4), 535-549. https://doi.org/10.2217/rme.14.20.

(14) Haryńska, A.; Gubanska, I.; Kucinska-Lipka, J.; Janik, H. Fabrication and Characterization of Flexible Medical-Grade TPU Filament for Fused Deposition Modeling 3DP Technology. Polymers (Basel). 2018, 10 (12), 1304. https://doi.org/10.3390/polym10121304.

(15) Harris, C. G.; Jursik, N. J. S.; Rochefort, W. E.; Walker, T. W. Additive Manufacturing With Soft TPU - Adhesion Strength in Multimaterial Flexible Joints. Front. Mech. Eng. 2019, 5. https://doi.org/10.3389/fmech.2019.00037.

(16) Tappa, K.; Jammalamadaka, U. Novel Biomaterials Used in Medical 3D Printing Techniques. J. Funct. Biomater. 2018, 9 (1), 17. https://doi.org/10.3390/jfb9010017.

(17) Lin, W.-C.; Yu, D.-G.; Yang, M.-C. Blood Compatibility of Thermoplastic Polyurethane 
Membrane Immobilized with Water-Soluble Chitosan/Dextran Sulfate. Colloids Surfaces B Biointerfaces 2005, 44 (2-3), 82-92. https://doi.org/10.1016/j.colsurfb.2005.05.015.

(18) Ehler, E.; Sterling, D.; Dusenbery, K.; Lawrence, J. Workload Implications for Clinic Workflow with Implementation of Three-Dimensional Printed Customized Bolus for Radiation Therapy: A Pilot Study. PLoS One 2018, 13 (10), e0204944. https://doi.org/10.1371/journal.pone.0204944.

(19) Dancewicz, O. L.; Sylvander, S. R.; Markwell, T. S.; Crowe, S. B.; Trapp, J. V. Radiological Properties of 3D Printed Materials in Kilovoltage and Megavoltage Photon Beams. Phys. Medica 2017, 38, 111-118. https://doi.org/10.1016/j.ejmp.2017.05.051.

(20) Madamesila, J.; McGeachy, P.; Villarreal Barajas, J. E.; Khan, R. Characterizing 3D Printing in the Fabrication of Variable Density Phantoms for Quality Assurance of Radiotherapy. Phys. Medica 2016, 32 (1), 242-247. https://doi.org/10.1016/j.ejmp.2015.09.013.

(21) Huang, J.-J.; Ren, J.-A.; Wang, G.-F.; Li, Z.-A.; Wu, X.-W.; Ren, H.-J.; Liu, S. 3D-Printed "Fistula Stent" Designed for Management of Enterocutaneous Fistula: An Advanced Strategy. World J. Gastroenterol. 2017, 23 (41), 7489-7494. https://doi.org/10.3748/wjg.v23.i41.7489.

(22) Mathew, E.; Domínguez-Robles, J.; Larrañeta, E.; Lamprou, D. A. Fused Deposition Modelling as a Potential Tool for Antimicrobial Dialysis Catheters Manufacturing: New Trends vs. Conventional Approaches. Coatings 2019, 9 (8), 515. https://doi.org/10.3390/coatings9080515.

(23) Weisman, J. A.; Nicholson, J. C.; Tappa, K.; Jammalamadaka, U.; Wilson, C. G.; Mills, D. K. Antibiotic and Chemotherapeutic Enhanced Three-Dimensional Printer Filaments and Constructs for Biomedical Applications. Int. J. Nanomedicine 2015, 10, 357-370. https://doi.org/10.2147/IJN.S74811.

(24) Weisman, J. A.; Ballard, D. H.; Jammalamadaka, U.; Tappa, K.; Sumerel, J.; D'Agostino, H. B.; Mills, D. K.; Woodard, P. K. 3D Printed Antibiotic and Chemotherapeutic Eluting Catheters for Potential Use in Interventional Radiology. Acad. Radiol. 2019, 26 (2), 270274. https://doi.org/10.1016/j.acra.2018.03.022.

(25) Gosbell, I. B. Diagnosis and Management of Catheter-Related Bloodstream Infections Due to Staphylococcus Aureus. Intern. Med. J. 2005, 35 (s2), S45-S62. https://doi.org/10.1111/j.1444-0903.2005.00979.x. 
(26) Thompson, S.; Wiebe, N.; Klarenbach, S.; Pelletier, R.; Hemmelgarn, B. R.; Gill, J. S.; Manns, B. J.; Tonelli, M. Catheter-Related Blood Stream Infections in Hemodialysis Patients: A Prospective Cohort Study. BMC Nephrol. 2017, 18 (1), 357. https://doi.org/10.1186/s12882-017-0773-5.

(27) Kerr, M. Chronic Kidney Disease in England: The Human and Financial Cost; 2012.

(28) Facts and Stats, Kidney care UK https://www.kidneycareuk.org/news-andcampaigns/facts-and-stats/ (accessed Mar 7, 2019).

(29) Sehmi, S. K.; Noimark, S.; Weiner, J.; Allan, E.; MacRobert, A. J.; Parkin, I. P. Potent Antibacterial Activity of Copper Embedded into Silicone and Polyurethane. ACS Appl. Mater. Interfaces 2015, 7 (41), 22807-22813. https://doi.org/10.1021/acsami.5b08665.

(30) Puperi, D. S.; Kishan, A.; Punske, Z. E.; Wu, Y.; Cosgriff-Hernandez, E.; West, J. L.; GrandeAllen, K. J. Electrospun Polyurethane and Hydrogel Composite Scaffolds as Biomechanical Mimics for Aortic Valve Tissue Engineering. ACS Biomater. Sci. Eng. 2016, 2 (9), 15461558. https://doi.org/10.1021/acsbiomaterials.6b00309.

(31) Arefin, A.; Mcculloch, Q.; Martinez, R.; Martin, S. A.; Singh, R.; Ishak, O. M.; Higgins, E. M.; Haffey, K. E.; Huang, J.-H.; lyer, S.; et al. Micromachining of Polyurethane Membranes for Tissue Engineering Applications. ACS Biomater. Sci. Eng. 2018, 4 (10), 3522-3533. https://doi.org/10.1021/acsbiomaterials.8b00578.

(32) Stewart, S.; Domínguez-Robles, J.; Donnelly, R.; Larrañeta, E. Implantable Polymeric Drug Delivery Devices: Classification, Manufacture, Materials, and Clinical Applications. Polymers (Basel). 2018, 10 (12), 1379. https://doi.org/10.3390/polym10121379.

(33) Baumann, F.; Bugdayci, H.; Grunert, J.; Keller, F.; Roller, D. Influence of Slicing Tools on Quality of 3D Printed Parts. Comput. Aided. Des. Appl. 2016, 13 (1), 14-31. https://doi.org/10.1080/16864360.2015.1059184.

(34) Larrañeta, E.; Henry, M.; Irwin, N. J.; Trotter, J.; Perminova, A. A.; Donnelly, R. F. Synthesis and Characterization of Hyaluronic Acid Hydrogels Crosslinked Using a Solvent-Free Process for Potential Biomedical Applications. Carbohydr. Polym. 2018, 181, 1194-1205. https://doi.org/10.1016/j.carbpol.2017.12.015.

(35) McCoy, C. P.; Irwin, N. J.; Brady, C.; Jones, D. S.; Carson, L.; Andrews, G. P.; Gorman, S. P. An Infection-Responsive Approach To Reduce Bacterial Adhesion in Urinary Biomaterials.
Mol.
Pharm.
2016,
13
(8),
2817-2822. 
https://doi.org/10.1021/acs.molpharmaceut.6b00402.

(36) Wang, R.; Neoh, K. G.; Shi, Z.; Kang, E.-T.; Tambyah, P. A.; Chiong, E. Inhibition of Escherichia Coli and Proteus Mirabilis Adhesion and Biofilm Formation on Medical Grade Silicone Surface. Biotechnol. Bioeng. 2012, 109 (2), 336-345. https://doi.org/10.1002/bit.23342.

(37) Jones, D. S.; McGovern, J. G.; Woolfson, A. D.; Gorman, S. P. Role of Physiological Conditions in the Oropharynx on the Adherence of Respiratory Bacterial Isolates to Endotracheal Tube Poly(Vinyl Chloride). Biomaterials 1997, 18 (6), 503-510. https://doi.org/10.1016/S0142-9612(96)00170-6.

(38) Miles, A. A.; Misra, S. S.; Irwin, J. O. The Estimation of the Bactericidal Power of the Blood. J Hyg 1938, 38, 732-749.

(39) Elastollan Thermoplastic Polyurethane https://aerospace.basf.com/elastollan.html (accessed Mar 8, 2019).

(40) Chopra, I.; Roberts, M. Tetracycline Antibiotics: Mode of Action, Applications, Molecular Biology, and Epidemiology of Bacterial Resistance. Microbiol. Mol. Biol. Rev. 2001, 65 (2), 232-260. https://doi.org/10.1128/MMBR.65.2.232-260.2001.

(41) Okoli, C. P.; Ofomaja, A. E. Development of Sustainable Magnetic Polyurethane Polymer Nanocomposite for Abatement of Tetracycline Antibiotics Aqueous Pollution: Response Surface Methodology and Adsorption Dynamics. J. Clean. Prod. 2019, 217, 42-55. https://doi.org/10.1016/j.jclepro.2019.01.157.

(42) Christ, J. F.; Aliheidari, N.; Ameli, A.; Pötschke, P. 3D Printed Highly Elastic Strain Sensors of Multiwalled Carbon Nanotube/Thermoplastic Polyurethane Nanocomposites. Mater. Des. 2017, 131, 394-401. https://doi.org/10.1016/j.matdes.2017.06.011.

(43) Jiang, L.; Su, C.; Ye, S.; Wu, J.; Zhu, Z.; Wen, Y.; Zhang, R.; Shao, W. Synergistic Antibacterial Effect of Tetracycline Hydrochloride Loaded Functionalized Graphene Oxide Nanostructures. Nanotechnology 2018, 29 (50), 505102. https://doi.org/10.1088/13616528/aae424.

(44) Naik, A. D.; Fontaine, G.; Bellayer, S.; Bourbigot, S. Salen Based Schiff Bases to Flame Retard Thermoplastic Polyurethane Mimicking Operational Strategies of Thermosetting Resin. RSC Adv. 2015, 5 (60), 48224-48235. https://doi.org/10.1039/C5RA06242J.

(45) Law, K.-Y. Definitions for Hydrophilicity, Hydrophobicity, and Superhydrophobicity: 
Getting the Basics Right. J. Phys. Chem. Lett. 2014, 5 (4), 686-688. https://doi.org/10.1021/jz402762h.

(46) Maki, D. G.; Cobb, L.; Garman, J. K.; Shapiro, J. M.; Ringer, M.; Helgerson, R. B. An Attachable Silver-Impregnated Cuff for Prevention of Infection with Central Venous Catheters: A Prospective Randomized Multicenter Trial. Am. J. Med. 1988, 85 (3), 307314. https://doi.org/10.1016/0002-9343(88)90579-7.

(47) Hasaniya, N. W. M. A.; Angelis, M.; Brown, M. R.; Yu, M. Efficacy of Subcutaneous SilverImpregnated Cuffs in Preventing Central Venous Catheter Infections. Chest 1996, 109 (4), 1030-1032. https://doi.org/10.1378/chest.109.4.1030.

(48) Chen, Y.; Dai, A.; Shi, Y.; Liu, Z.; Gong, M.; Yin, X. Effectiveness of Silver-Impregnated Central Venous Catheters for Preventing Catheter-Related Blood Stream Infections : A Meta-Analysis. Int. J. Infect. Dis. 2014, 29, 279-286. https://doi.org/10.1016/j.ijid.2014.09.018.

(49) Galloway, S.; Bodenham, A. Long-Term Central Venous Access. Br. J. Anaesth. 2004, 92 (5), 722-734. https://doi.org/10.1093/bja/aeh109.

(50) Moretti, E. W.; Ofstead, C. L.; Kristy, R. M.; Wetzler, H. P. Impact of Central Venous Catheter Type and Methods on Catheter-Related Colonization and Bacteraemia. J. Hosp. Infect. 2005, 61 (2), 139-145. https://doi.org/10.1016/j.jhin.2005.02.012.

(51) Scott-Warren, V.; Morley, R. Paediatric Vascular Access. BJA Educ. 2015, 15 (4), 199-206. https://doi.org/10.1093/bjaceaccp/mku050.

(52) Liu, Z.; Wang, Y.; Wu, B.; Cui, C.; Guo, Y.; Yan, C. A Critical Review of Fused Deposition Modeling 3D Printing Technology in Manufacturing Polylactic Acid Parts. Int. J. Adv. Manuf. Technol. 2019, 102 (9-12), 2877-2889. https://doi.org/10.1007/s00170-01903332-x.

(53) Lalehpour, A.; Barari, A. Post Processing for Fused Deposition Modeling Parts with Acetone Vapour Bath. IFAC-PapersOnLine 2016, 49 (31), 42-48. https://doi.org/10.1016/j.ifacol.2016.12.159.

(54) Zhao, G.; Ma, G.; Feng, J.; Xiao, W. Nonplanar Slicing and Path Generation Methods for Robotic Additive Manufacturing. Int. J. Adv. Manuf. Technol. 2018, 96 (9-12), 3149-3159. https://doi.org/10.1007/s00170-018-1772-9.

(55) Alhers, D. 3D Printing of Nonplanar Layersfor Smooth Surface Generation, Universität 
Hamburg, 2018.

(56) Macha, I. J.; Ben-Nissan, B.; Vilchevskaya, E. N.; Morozova, A. S.; Abali, B. E.; Müller, W. H.; Rickert, W. Drug Delivery From Polymer-Based Nanopharmaceuticals-An Experimental Study Complemented by Simulations of Selected Diffusion Processes. Front. Bioeng. Biotechnol. 2019, 7. https://doi.org/10.3389/fbioe.2019.00037.

(57) Kaur, G.; Grewal, J.; Jyoti, K.; Jain, U. K.; Chandra, R.; Madan, J. Oral Controlled and Sustained Drug Delivery Systems. In Drug Targeting and Stimuli Sensitive Drug Delivery Systems; Elsevier, 2018; pp 567-626. https://doi.org/10.1016/B978-0-12-8136898.00015-X.

(58) Goyanes, A.; Fina, F.; Martorana, A.; Sedough, D.; Gaisford, S.; Basit, A. W. Development of Modified Release 3D Printed Tablets (Printlets) with Pharmaceutical Excipients Using Additive Manufacturing. Int. J. Pharm. 2017, 527 (1-2), 21-30. https://doi.org/10.1016/j.ijpharm.2017.05.021.

(59) Genina, N.; Holländer, J.; Jukarainen, H.; Mäkilä, E.; Salonen, J.; Sandler, N. Ethylene Vinyl Acetate (EVA) as a New Drug Carrier for 3D Printed Medical Drug Delivery Devices. Eur. J. Pharm. Sci. 2016, 90, 53-63. https://doi.org/10.1016/j.ejps.2015.11.005.

(60) Larrañeta, E.; Imízcoz, M.; Toh, J. X.; Irwin, N. J.; Ripolin, A.; Perminova, A.; DomínguezRobles, J.; Rodríguez, A.; Donnelly, R. F. Synthesis and Characterization of Lignin Hydrogels for Potential Applications as Drug Eluting Antimicrobial Coatings for Medical Materials. ACS Sustain. Chem. Eng. 2018, 6 (7), 9037-9046. https://doi.org/10.1021/acssuschemeng.8b01371.

(61) Tong, S. Y. C.; Davis, J. S.; Eichenberger, E.; Holland, T. L.; Fowler, V. G. Staphylococcus Aureus Infections: Epidemiology, Pathophysiology, Clinical Manifestations, and Management. Clin. Microbiol. Rev. 2015, 28 (3), 603-661. https://doi.org/10.1128/CMR.00134-14.

(62) Khan, H. A.; Baig, F. K.; Mehboob, R. Nosocomial Infections: Epidemiology, Prevention, Control and Surveillance. Asian Pac. J. Trop. Biomed. 2017, 7 (5), 478-482. https://doi.org/10.1016/j.apjtb.2017.01.019.

(63) CDC. Types of healthcare-associated infections. Healthcare-associated infections (HAIs) https://www.cdc.gov/HAl/infectionTypes.html (accessed Mar 10, 2019).

(64) Sydnor, E. R. M.; Perl, T. M. Hospital Epidemiology and Infection Control in Acute-Care 
Settings. Clin. Microbiol. Rev. 2011, 24 (1), 141-173. https://doi.org/10.1128/CMR.00027-10.

(65) Hall, C. W.; Mah, T.-F. Molecular Mechanisms of Biofilm-Based Antibiotic Resistance and Tolerance in Pathogenic Bacteria. FEMS Microbiol. Rev. 2017, 41 (3), 276-301. https://doi.org/10.1093/femsre/fux010.

(66) Statement by FDA Commissioner Scott Gottlieb, M.D., on FDA ushering in new era of 3D printing of medical products; provides guidance to manufacturers of medical devices https://www.fda.gov/news-events/press-announcements/statement-fdacommissioner-scott-gottlieb-md-fda-ushering-new-era-3d-printing-medical-products (accessed Mar 15, 2019). 